\title{
Radiology at the Intersection: We have met the Enemy and He is Us!
}

\section{Shraddha K Singhania}

How to cite this article: Singhania SK. Radiology at the Intersection: We have met the Enemy and $\mathrm{He}$ is Us! Int J Recent Surg Med Sci 2017;3(1):1-2.

\section{Source of support: Nil}

\section{Conflict of interest: None}

I was thinking of events in the development of radiology from the time when my older generation used to be radiologists and was reminded of a humorous phrase by Walt Kelly: "We have met the enemy and he is us!" There is a speedy development of newer imaging techniques and introduction of newer applications at an equal velocity.

Initially, the radiologists were everywhere, and were in constant touch with the referring physicians, who visited the radiology department to view images, discuss cases, rule out differential diagnosis and reach a conclusion. But now, however, with technologic changes, like introduction of picture archiving and communication systems (PACS), there has been an intercession with this long-standing tradition in a way that it may put us radiologists back in the clinical field if we are not careful. Previously, only the specialist neurologist, neurosurgeon, or oncosurgeon relied on their knowledge of the subject to reach the diagnosis. But now with availability of the radiological equipments in every field, we have come to a situation where the subspecialist physicians have also started interpreting imaging on their own and acting as primary providers. And if the radiologist continues reporting imaging only about signal densities and general findings and advising "please correlate clinically" phrase for the physician, radiology runs the risk of being pushed to the background and everything getting correlated clinically.

By rapid advances in electronic communications, PACS is proving to be an ingenious innovation. It has allowed almost instant availability of images to everybody

\section{Assistant Lecturer}

Department of Radiology, Datta Meghe Institute of Medical Sciences, Wardha, Maharashtra, India

Corresponding Author: Shraddha K Singhania, Assistant Lecturer, Department of Radiology, Datta Meghe Institute of Medical Sciences, Wardha, Maharashtra, India, e-mail: drshraddha singhania@hotmail.com and of reports which has helped speeding up patient care and has eliminated dark room hazards, but it has also brought along some serious disadvantages. Radiologists are now generally reporting from their offices rather from the department or the imaging rooms; as a result, patients

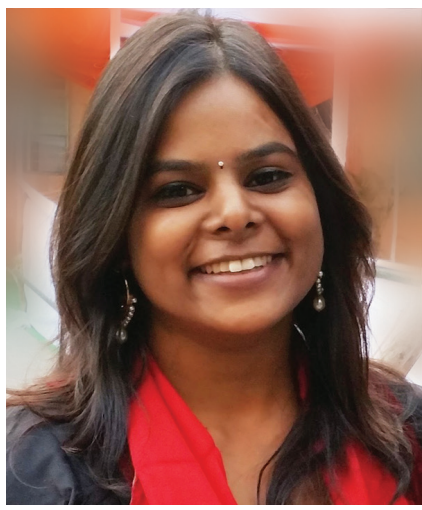
never interact with the radi-

ologist except when undergoing a radiological procedure or during ultrasonography or mammogram, which these days too is done by the technicians. There is a protocol of requirement of radiological help to be provided during emergencies by faculty and residents; however, the images are increasingly being sent over the Internet to remote sites, where radiologists read them without any personal contact with treating physicians and generally with little or no clinical information. These developments threaten to change radiology into a commodity and radiologists, in patients' eyes, into nonparticipants in their care. Many patients do not know that the radiologists are physicians who play an important role in their care or they do not even know that radiology is a branch of medicine.

Since radiology is an indispensible part of hospitals, measures to facilitate the use of diagnostics is being undertaken these days like establishment of superspecialty subdepartments and perseverance toward researches and original studies. The public should be made aware of the clinical role of radiology, and radiologists' contact with the patients should be increased. The radiologists of today should standardize the reporting formats used in different organ systems, which can help in communication and second opinion between fellow radiologists and the clinicians. Another place where generally the radiologists do not have a say is at the tumor board. Subspecialty trainees should participate in clinical rounds, clinicoradiological and tumor board meetings. If radiologists need to walk hand in hand with the clinician, they need to subspecialize, and work further rather than just screening the patient. If they feel that the patient requires further investigations, they should get them done and take part in complete analysis. 
Referring physicians will initially resist many of these changes just as anyone resists an initial change, but, ultimately, these changes will be accepted and embraced, as have many others since Roentgen's days, and our coming generation will return to playing their full roles as radiophysicians. They will not only sit in front of PACS reporting but will also see patients and remain in touch with their treating clinicians. This is how and why our specialty was started and as it has advanced, it has consistently responded to the times change, and it will flourish in future. The saying "times change" is as true today as it was in the days of the dark room.

As Jimmy Carter once said, We must adjust to changing times but still hold to unchanging principles. 